\title{
El transporte de carga y el comercio internacional de los países miembros del TLCAN: Un análisis de elasticidades
}

\author{
Freight transport and international trade of NAFTA member: \\ An elasticity analysis
}

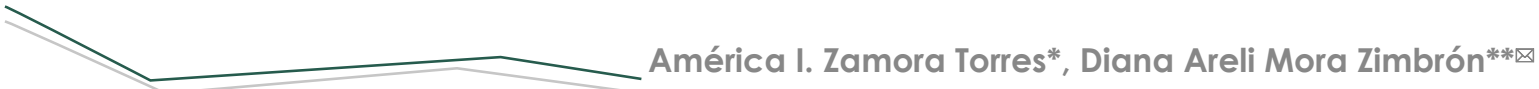

Zamora Torres, A. I., \& Mora Zimbrón, D. A. (2018). El transporte de carga y el comercio internacional de los países miembros del TLCAN: Un análisis de elasticidades. Investigación y Ciencia de la Universidad Autónoma de Aguascalientes, 26(74), 58-72.
\end{abstract}

\section{RESUMEN}

El presente trabajo aborda el tema del transporte de carga en el contexto del comercio internacional entre los países miembros del Tratado de Libre Comercio de América del Norte; es decir, México, Estados Unidos y Canadá mediante un análisis de elasticidades con las variables: infraestructura, costos, importaciones, exportaciones y volumen de carga en el transporte carretero. La importancia del tema radica en la sustancial operativa comercial entre los países miembros del TLCAN, que ha provocado una significativa dependencia de la economía mexicana a partir de la firma del mismo. Entre los principales resultados se muestra que las exportaciones mexicanas hacia EE. UU. presentan una elasticidad positiva con relación a la infraestructura, se puede afirmar que los cambios en la red carretera provocan una mayor sensibilidad en el comportamiento de las ex-

Palabras clave: elasticidades; México; Estados Unidos; Canadá; transporte carretero.

Keywords: elasticities; Mexico; United States; Canada; road transport.

\section{Recibido: 31 de agosto de 2017, aceptado: 5 de marzo de 2018}

* Instituto de Investigaciones Económicas y Empresariales, Universidad Michoacana de San Nicolás de Hidalgo. Gral. Francisco J. Múgica s/n, Felicitas del Río, C. P. 58040, Morelia, Michoacán, México. Correo electrónico: americazł@gmail.com

** Escuela de Negocios Internacionales, Universidad de Morelia. Fray Antonio de Lisboa 22, Col. 5 de Mayo, C. P. 58230, Morelia, Michoacán, México.

Correo electrónico: dianazimbron@hotmail.com

Autor para correspondencia portaciones. Las elasticidades obtenidas para las importaciones mexicanas provenientes de EE. UU. con respecto a la infraestructura fueron elásticas positivas, con excepción de los años 2001 y 2009.

\section{ABSTRACT}

The present paper proposes to address the issue of freight transportation in the context of international trade between the member countries of the North American Free Trade Agreement, that is, between Mexico, the United States and Canada through an analysis of elasticities and variables considered: infrastructure, costs, imports, exports and cargo volume in road transport. The importance of the present subject is that there is an important commercial operation between the three countries members of NAFTA and this commercial operation has provoked a significant dependence on the part of the Mexican economy from the signature of the same one. Among the main results it is shown that mexican exports to the US in relation to infrastructure in most positive elasticities results, it is possible to review the changes in the road network cause a greater sensitivity in the behavior of exports. Regarding the elasticities obtained for mexican imports from the United States with respect to the infrastructure of elastic tests, with the exception of the years 2001 and 2009. 
INTRODUCCIÓN

México cuenta con 12 tratados de libre comercio, de los cuales, el TLCAN ha sobresalido económica y mercadológicamente, desde su firma y posterior entrada en vigor, el 1 de diciembre de 1994. El TLCAN cobra importancia debido a la frontera compartida con EE. UU. y por ser con este país con el que México mantiene el mayor porcentaje de sus relaciones comerciales internacionales.

De acuerdo con la Secretaría de Economía (2014), el objetivo de este tratado es "formar una Zona de Libre Comercio, estableciendo reglas claras y permanentes para el intercambio comercial, que permita el incremento de flujo comercial e inversión, así como nuevas oportunidades de empleo y mejores niveles de vida" (pág. cita textual).

Las cifras publicadas por el Instituto Nacional de Estadística y Geografía con relación al comercio exterior de México con Canadá y EE. UU. muestran que del total de las exportaciones mexicanas durante $2015,81.5 \%$ y $2.77 \%$ tuvieron como destino EE. UU. y Canadá, respectivamente; mientras para las importaciones mexicanas totales para el mismo año el INEGI reportó que $47.26 \%$ fueron de procedencia estadounidense y $2.52 \%$ canadiense.

El comercio estadounidense hacia México ha cambiado desde 1994. De acuerdo con información publicada por la Oficina del Censo de los EE. UU. (US Census Bureau, 2015), las importaciones estadounidenses procedentes de México en 1994 eran de $9.85 \%$, mientras del total de sus exportaciones, $10.12 \%$ eran enviadas hacia México. Al 2015, dichas cifras eran de $19.63 \%$ y $15.61 \%$, respectivamente. Es notable el aumento en el comercio estadounidense con México, aunque evidente también la dependencia comercial de México con respecto a la economía norteamericana. Sin embargo, EE.UU. ha sabido diversificar sus operaciones comerciales con otros países.

En cuanto al tipo de transporte utilizado, México tiene una gran preferencia por la utilización del transporte terrestre para su operativa comercial. De dicho sector, el autotransporte de carga o transporte carretero es el más utilizado, sobre todo en el comercio exterior mexicano, debido al bajo costo del mismo, el fácil acceso a empresas dedicadas a este giro para el transporte de mercancías y, sobre todo, debido a la cercanía con EE. UU., situación por la cual las empresas prefieren enviar sus productos por medio del autotransporte.

De acuerdo con datos del INEGI, en relación con los bienes exportados e importados por México en 2013, el transporte carretero fue el que más mercancía operó, con $56 \%$ y $51 \%$ de exportaciones e importaciones, respectivamente. Tanto para Canadá como para México el transporte carretero es el más utilizado en materia de comercio exterior con $43 \%$ y $54 \%$, respectivamente, según el INEGI (2012), lo que implica solamente una de las similitudes existentes entre ambos países. La norma operativa del transporte de carga ha sido variable desde antes de la firma del tratado. En la década de los 80 los camiones de carga domiciliados en México podían solicitar una autorización a la Comisión Interestatal de Comercio para acceder y operar en EE. UU.

No obstante, en 1982 el Congreso estadounidense autorizó la iniciativa presidencial consistente en imponer una moratoria de dos años para la expedición de nuevos permisos que autorizaran la operación de los camiones de carga con domicilio en los países vecinos mediante la Ley de Reforma de la Regulación de Transporte, por lo que las unidades mexicanas y canadienses quedaron con acceso restringido al territorio estadounidense, como respuesta México y Canadá negaron también el acceso de camiones estadounidenses a sus respectivos países (Mendoza Cota \& Díaz, 2003).

Para Canadá este conflicto no duró mucho tiempo; sin embargo, a México le llevó más de una década avanzar en el tema; es decir, hasta la firma del TLCAN en 1994, ya que el anexo del TLCAN que hace referencia al transporte de carga carretero establece que EE. UU. deberá levantar gradualmente la moratoria a los camiones domiciliados en México para su operación dentro de territorio estadounidense, por lo que a partir de la entrada en vigor del tratado EE. UU. comenzó a admitir solicitudes de autobuses mexicanos de pasajeros con el objetivo de conducir unidades bajo la operación de contratos de flete e incluso para realizar recorridos turísticos en EE. UU. (SCT, 2014).

En este contexto, las empresas que proporcionan servicios de carga vieron la oportunidad de negociar en un mercado cuyo dinamismo económico fuera mayor. Lamentablemente, dicha situación fue frenada por el sindicato de camioneros en EE. UU., denominado The International Brotherhood of Team- 
IIVESTIGACIÓn Y CIERCIA DE LA UNIVERSIDAD AUTÓNOMA DE AGUASCALIERTES sters, que solicitaron la suspensión de la Regla de la Comisión en la Corte de Apelaciones de EE. UU. en el Distrito de Columbia el 15 de diciembre de 1995. Tres días después se anunció la suspensión indefinida de la aplicación de las disposiciones del TLCAN para el acceso de los camiones de carga por parte del Departamento de Transporte de EE. UU. (Mendoza Cota \& Díaz, 2003). Las empresas transportistas en México regresaban a la situación de la década de los 80, aun con la firma del tratado más anunciado en la historia mexicana.

En el año 2000 un panel de arbitraje formado por miembros de ambos países admitió la violación del TLCAN ante la suspensión impuesta por EE.UU. a los camiones mexicanos (SCT, 2014). Sin embargo, el gobierno mexicano abandonó el tema del transporte de carga por 10 años, aun con el fallo a favor del panel de arbitraje. Fue hasta el mes de marzo de 2011 que la SCT (2015), en conjunto con el Departamento de Transporte de EE. UU., suscribió un memorándum donde una vez cumplidos una serie de procedimientos, se permitía a las empresas tanto mexicanas como estadounidenses el tránsito permanente en ambos países, como respuesta al programa piloto de autotransporte transfronterizo entre México y EE. UU., cuya duración fue de tres años (Mendoza Cota \& Díaz, 2003). Las empresas que finalizaron el programa piloto obtuvieron, a principios de 2016, el permiso de prestación de Servicios Internacionales de Autotransporte de Carga de Largo Recorrido, dos décadas después de la firma del TLCAN.

El objetivo del presente estudio fue realizar un análisis del transporte de carga internacional mexicano mediante la determinación de las principales variables que inciden en el comportamiento del comercio internacional de los países miembros del TLCAN.

Con el fin de lograr el objetivo planteado se propuso un estudio de elasticidades partiendo del concepto de elasticidad desde la perspectiva económica, término introducido por el matemático, filósofo y economista francés Antoine-Auguste Cournot como una medida de la sensibilidad de la cantidad demandada o de la cantidad ofrecida ante el cambio en alguno de sus factores determinantes, es utilizada generalmente con respecto al precio o la renta (Silvestre Méndez, 1999).

Alfred Marshall en 1890 consolida el concepto mediante la elasticidad precio de la demanda; es decir, mediante la utilización de las variables oferta y demanda, con la finalidad de estimar la sensibilidad que muestra la cantidad obtenida ante los pequeños cambios registrados por el precio (Marshall en Silvestre Méndez, 1999). No obstante, el cálculo de elasticidades es de gran utilidad debido a que permite un manejo de diferentes variables, no solo el precio y la demanda como fue desarrollado en sus orígenes.

Este trabajo se divide en cinco secciones -incluida introducción- donde se describe la situación y comportamiento del transporte de carga internacional en México, EE. UU. y Canadá, seguida de la sección de Materiales y Métodos, describiendo el procedimiento metodológico a utilizar, posteriormente se mencionan los resultados obtenidos y se realiza un breve análisis de los mismos en la parte de discusión, por último en la sexta sección las principales conclusiones alcanzadas, así como la discusión de las mismas.

\section{MATERIALES Y MÉTODOS}

La mayor parte de los estudios relativos al tema del transporte de carga enfatizan la importancia de los costos y la infraestructura. De acuerdo al IMT (2015), los costos de operación en el transporte en este sector son muy variables debido a que están en función del mantenimiento de la unidad, la forma de conducción de cada operador, los tipos de caminos, el nivel de utilización, tipo de motores, antigüedad de las unidades, entre otras características. Por tanto, cada unidad tiene su costo de operación, que no es considerado de manera precisa por los transportistas, sobre todo por los pequeños proveedores denominados hombres-camión, que coexisten con empresas de mayor capacidad.

Dussel Peters (2008) también refiere los costos al plantear la realización de un estudio de mediano y largo plazo del transporte terrestre de carga, tanto a nivel regional como sectorial. Mientras, Coase (1988) define los costos de transacción como: "los costos de medir el valor de los atributos de lo que se está intercambiando y los costos de proteger derechos, vigilar y hacer valer acuerdos", aunque es importante tomar en cuenta que en México las empresas transportistas y los hombres-camión calculan sus costos de formas diferentes, por lo que no existe unificación en el cálculo de estos. 
Sáez y Valdés (1999) se enfocan en la importancia de los acuerdos para lograr una reducción en los costos debido a que los acuerdos se encargan de proveer un conjunto de reglas aplicables a los miembros, que proporcionan estabilidad, estimulan la transparencia mediante obligaciones específicas y promueven su cumplimiento mediante instrumentos vinculantes. De acuerdo con la SCT (2014), el autotransporte mexicano opera $80 \%$ de la carga por medio de unidades motrices, utilizando mayormente tractocamiones de tres y dos ejes, camiones de dos y tres ejes, entre otros; de los cuales no todos se encuentran registrados actualmente ante la SCT, sin mencionar unidades de arrastre y grúas industriales.

Otra de las variables que más enfatizan las investigaciones relativas al transporte de carga en el comercio internacional es la infraestructura. Prud'homme (2005) compara el efecto de la infraestructura y la reducción de aranceles con el tamaño del mercado, afirmando que ante una inversión en infraestructura se provoca una reducción de aquellos y un incremento en el tamaño del mercado en una determinada economía. Asimismo, Aschaver (1989) afirma que la infraestructura tiene un efecto positivo en la producción, por lo que la productividad se ve afectada de manera directa por la infraestructura en el transporte, y abarca puertos, carreteras y aeropuertos. Cipoletta Tomassian, Pérez Salas y Sánchez (2010) resaltan la influencia que tienen las políticas sectoriales y los aspectos medioambientales en el transporte y en la logística, sin dejar de mencionar la variable infraestructura con acento en la importancia de la calidad y la utilización de la misma; mientras que Auschaver (1989), Garcia-Milà \& McGuire (1992) y Munell (1990) afirman que un aumento en la red carretera tiene un efecto positivo en el crecimiento del PIB per cápita.

La calidad en la infraestructura es una variable primordial en el estudio de la eficiencia del transporte, aparece también citada en estudios realizados por Wilmsmeier y Sánchez (2009) y Arvis, Alina Mustra, Panzer, Ojala, \& Naula (2007), entre otros. Existen otras variables que determinan las exportaciones, como el tipo de cambio real que de acuerdo con Macías (2003) es una de las variables más importantes en la operativa comercial de un país con el exterior, así como los costos laborales en el sector en cuestión, ya que la dinámica laboral es diferente. En México un conductor de camión percibe alrededor de 11,691 dólares estadounidenses al año (Wagelndicator Foundation, 2016), mientras un conductor estadounidense contratado por una empresa privada percibe 44,000 dólares estadounidenses anuales según el US Department of Labor (Departamento de Trabajo de los Estados Unidos, 2016).

Si se toma como base la revisión de estudios comentados se propone el cálculo de elasticidades puntuales, utilizando como datos principales la operativa comercial de los tres países que conforman el TLCAN con respecto a la economía mexicana, en relación con las dos variables destacadas en la mayoría de los estudios relativos al transporte; es decir, la infraestructura y los costos. Estos últimos representados por el índice de precios al productor debido a la variabilidad del cálculo entre una empresa y un hombre-camión en sus costos, una de las razones por las que México actualmente no cuenta con una base estadística relativa a los costos de transporte.

La elasticidad de manera aritmética se representa mediante el cambio porcentual de la cantidad demandada dividida entre el cambio porcentual en el precio, en donde hay una relación inversa (Gómez Leyja, \& Hernández Rodríguez, 1995). Se pueden encontrar de forma estricta dos tipos de sensibilidades entre variables en el cálculo de elasticidades: elástica e inelástica; ambos casos son los extremos de los posibles resultados que se pueden obtener al realizar dicho cálculo. Se afirma que se tiene una sensibilidad elástica cuando el valor de la elasticidad es mayor a uno. Por ejemplo, en la elasticidad precio de la demanda indica que a un determinado cambio porcentual en el precio le corresponde un cambio porcentual mayor en la cantidad demandada (Gómez Leyja \& Hernández Rodríguez, 1995). En la figura 1 se pueden observar las relaciones elásticas e inelásticas entre las variables precio y demanda.



Figura 1. Elasticidad elástica e inelástica. Elaboración propia. 
En relación con la demanda inelástica, ésta indica que la cantidad demandada es muy poco sensible a los cambios en el precio, en este caso menor a uno; por tanto, si el precio disminuyera la cantidad demandada aumentaría menos que proporcionalmente al cambio en el precio. En un escenario ideal se desprende el razonamiento de la elasticidad unitaria indicando que tanto el cambio porcentual del precio como el de la demanda son iguales, por lo que la cantidad demandada se mantiene constante a cualquier nivel de precio (Gómez Leyja \& Hernández Rodríguez, 1995). La elasticidad del precio de la demanda de un producto o servicio se refiere a la respuesta a las variaciones del precio, su objetivo es maximizar las ganancias (Gujarati \& Porter, 2010).

A grandes rasgos, la fórmula para el cálculo de una elasticidad es la siguiente:

Elasticidad precio de la demanda $=\frac{\text { Porcentaje cambio de demanda }}{\text { Porcentaje cambio precio }}$

La elasticidad de la demanda de servicios del autotransporte de carga mexicano en el comercio internacional con respecto a la infraestructura logística indica el cambio porcentual de la demanda en respuesta a un cambio de $1 \%$, por ejemplo, en el total de la red carretera. Conocer el dato anterior beneficia y apoya a las empresas en la planeación de sus estrategias empresariales y al gobierno en materia de políticas públicas y apoyo empresarial. El objetivo de realizar el cálculo de elasticidades puntuales es medir y conocer la sensibilidad de una determinada variable $X$ en una variable $Y$, para determinar la influencia de las variables propuestas en relación con el autotransporte de carga mexicano con respecto a la operativa comercial que maneja con sus socios de norteamericanos; es decir, EE. UU. y Canadá. Las series de datos utilizadas corresponden a los años 1990-2014, hay que tomar en cuenta que el TLCAN fue firmado en 1992 y entró en vigor en 1994; las series fueron obtenidas de las Estadísticas de Transporte de América del Norte (2013) y el Instituto Nacional de Estadística y Geografía $(2012,2013)$.

Para representar el transporte de carga se utilizarán los datos relativos a las importaciones y exportaciones mexicanas realizadas con EE. UU. y Canadá mediante la utilización del transporte carretero de carga (tabla 1). Las variables independientes son el costo y la infraestructura; el costo se encuentra representado por el índice de precios al productor de transporte de carga, y la infraestructura por el total de la red carretera en México y las unidades vehiculares de carga mexicanas.

La elasticidad mide la sensibilidad de una variable con respecto a otra y es un número libre de medidas, debido a que es una razón entre cambios porcentuales, se puede representar de la siguiente manera:

$e=\frac{\% \Delta X}{\% \Delta Y} ; \quad X$ Variable dependiente; $\quad Y$ Variable independiente

Tabla 1

Variables utilizadas en el cálculo de elasticidades puntuales

\begin{tabular}{|c|c|}
\hline Transporte de carga (carretero) & Siglas \\
\hline Exportaciones a EE. UU.-Transporte carretero de carga & (EXP-EE. UU.) \\
\hline Exportaciones a Canadá-Transporte carretero de carga & (EXP-CANADA) \\
\hline Importaciones de EE. UU.-Transporte carretero de carga & (IMP-EE. UU.) \\
\hline Importaciones de Canadá-Transporte carretero de carga & (IMP-CANADA) \\
\hline Volumen de carga mexicano & (VC) \\
\hline \multicolumn{2}{|l|}{ Costos } \\
\hline Índice de precios al productor de transporte de carga & (INPPAC) \\
\hline \multicolumn{2}{|l|}{ Infraestructura } \\
\hline Carreteras total de la red & $(\mathrm{TRC})$ \\
\hline Unidades vehiculares de carga & (UV) \\
\hline
\end{tabular}




\section{InVESTIGACIÓn Y CIERCIA DE LA UחIVERSIDAD AUTÓnOMA DE RGUASCALIERTES}

Donde $e$ es el símbolo que representa la elasticidad; es decir, la sensibilidad que tiene la variable $X$ ante un cambio porcentual de la variable $Y$. Dicho en otras palabras: en qué porcentaje depende la variable $X$ de un movimiento porcentual de la variable $Y$. Existen diferentes tipos de elasticidades, clasificadas de acuerdo a si la elasticidad es mayor, igual o menor que la unidad: elásticas, unitarias o inelásticas, respectivamente.

Cuando la variable dependiente $X$ es relativamente insensible a las variaciones de la variable $Y$ la elasticidad toma valores menores a la unidad. De acuerdo con la condición de Marshall Lerner el resultado anterior indica que la variable $X$ no presenta ningún cambio cuando se presenta una variación en $Y$; es decir, que la presente relación de variables no presentaría influencia alguna en el comportamiento del autotransporte de carga relativo al comercio exterior con México o Canadá.

Por otra parte, cuando se obtiene una elasticidad unitaria, se entiende que la variable dependiente $X$ es sensible a las variaciones de $Y$, cabe resaltar que dicha variación no ocasiona un cambio relevante en el comportamiento de la variable $X$. Si la variable dependiente $X$ es muy sensible a las variaciones de $Y$, se obtiene como resultado una elasticidad mayor a la unidad. Dicho resultado, con referencia a la condición de Marshall-Lerner, significa que ante una variación porcentual de $Y$, la variable $X$ provoca un cambio significativo en el comportamiento del autotransporte de carga internacional mexicano (Mahmud, Ullah, \& Yucel, 2004).

Las clasificaciones de elasticidad mencionadas en los párrafos anteriores se pueden observar en la tabla 2.

Tabla 2

Clasificación de las elasticidades

\begin{tabular}{cc}
\hline Elasticidad & Clasificación \\
\hline$E>1$ & Elástica \\
$E=1$ & Elástica unitaria \\
$E<1$ & Inelástica \\
\hline
\end{tabular}

Nota: Elaboración propia.

Algunas de las elasticidades a calcular durante el presente ensayo se presentan a continuación, se refieren al comercio exterior mexicano con Canadá.

$$
\begin{gathered}
e=\frac{\% \Delta M \text { Canadá }}{\% \Delta I N P P A C} e=\frac{\% \Delta M \text { Canadá }}{\% \Delta T R C} e=\frac{\% \Delta M \text { Canadá }}{\% \Delta U V C} \\
e=\frac{\% \Delta X \text { Canadá }}{\% \Delta I N P P A C} e=\frac{\% \Delta X \text { Canadá }}{\% \Delta T R C} e=\frac{\% \Delta X \text { Canadá }}{\% \Delta U V C}
\end{gathered}
$$

Donde $M$ y $X$ son las variables dependientes y se refieren a las importaciones y exportaciones mexicanas a Canadá mediante la utilización del autotransporte de carga, respectivamente, mientras las variables independientes que se consideraron fueron: el Índice Nacional de Precios al Productor de transporte de carga (INPPAC); el total de la red carretera (TRC) y las unidades vehiculares de carga (UVC). También se calcularon las elasticidades referentes al comercio con EE. UU., donde $M$ y $X$ son las variables dependientes y se refieren a las importaciones y exportaciones mexicanas a EE. UU. mediante la utilización del autotransporte de carga, respectivamente, mientras las variables dependientes fueron las mismas que para el comercio con Canadá.

$$
\begin{array}{lll}
e=\frac{\% \triangle M E E U U}{\% \triangle I N P P A C} ; & e=\frac{\% \triangle M E E U U}{\% \triangle T R C} ; & e=\frac{\% \triangle M E E U U}{\% \triangle U V C} ; \\
e=\frac{\% \triangle X E E U U}{\% \triangle I N P P A C} ; & e=\frac{\% \triangle X E E U U}{\% \Delta T R C} ; & e=\frac{\% \triangle X E E U U}{\% \Delta U V C}
\end{array}
$$

Adicionalmente, se realizó el cálculo de las elasticidades referentes al volumen de carga mexicano, siendo ésta una de las variables dependientes, mientras las variables independientes son las mismas que en las elasticidades correspondientes a EE. UU. y Canadá. Se consideró importante tomar en cuenta las variaciones en el volumen de carga para conocer el comportamiento del mercado mexicano en relación con los cambios económicos locales e internacionales y, sobre todo, la evolución que se haya tenido a partir de la firma del TLCAN.

$$
e=\frac{\% \Delta V C}{\% \triangle I N P P A C} ; \quad e=\frac{\% \Delta V C}{\% \Delta T R C} ; \quad e=\frac{\% \Delta V C}{\% \Delta U V C}
$$

Por último, para el mismo periodo en cuestión se calculó un modelo de regresión simple mediante el método de mínimos cuadrados. Lo anterior, de acuerdo con Gujarati y Porter (2010) con el objetivo de confirmar la dependencia de una variable respecto a otra, con un nivel de significancia de $5 \%$, en donde a medida que el valor de la variable independiente incrementa en una unidad, el incremento estimado en la variable dependiente incrementará en promedio el valor que se obtenga en el coeficiente. 
IIVESTIGACIÓn Y CIERCIA DE LA UחIVERSIDAD AUTÓNOTH

64

\section{DE AGUASCALIERTES}

Dicho cálculo se llevó a cabo con ayuda del paquete estadístico Eviews 7.

\section{RESULTADOS}

A continuación se explican los resultados de las elasticidades obtenidas, en el detalle de las elasticidades de las importaciones mexicanas a Canadá mediante el transporte carretero con respecto al índice de precios al productor desde 1990, que se puede observar en la figura 2. Las variaciones comienzan a presentarse a partir de 1996 de manera positiva, para regresar aproximadamente al mismo nivel al año siguiente debido a que es precisamente en 1996 cuando las importaciones mexicanas provenientes de Canadá comenzaron a aumentar de manera constante, lo que se puede explicar por la crisis económica denominada burbuja puntocom, que a su vez marcó la rápida fundación de empresas que se encontraban vinculadas al internet, que en 2002 afectaron los mercados internacionales debido a su cierre o fusión.

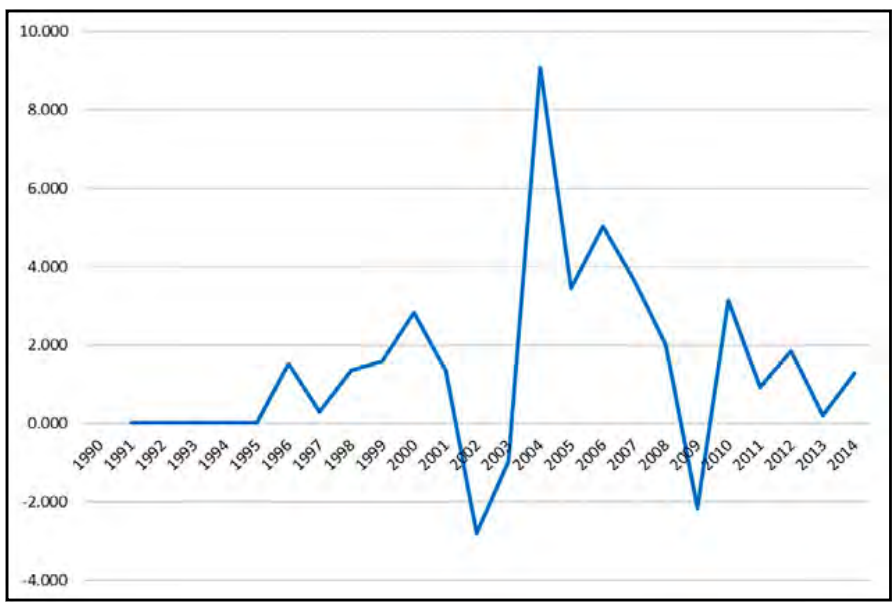

Figura 2. Elasticidades de las importaciones mexicanas provenientes de Canadá mediante el transporte carretero con respecto al índice de precios al productor.

Elaboración propia con base en datos del INEGI (2016) y Estadísticas de Transporte de América del Norte (2013).

En 2002 las importaciones provenientes de Canadá disminuyeron de manera drástica, en 2009 se dio una reducción aunque no del nivel presentado en 2002; lo que se puede explicar debido a la recesión en la que entró México en 2009 como consecuencia de la crisis que vivía EE. UU., donde México tuvo la mayor recesión de los países de América Latina, que incluso aumentó su deuda externa en $70 \%$. El comportamiento de las importaciones mexicanas provenientes de Canadá por medio del transporte carretero con respecto a la variable que representan a la infraestructura se puede observar en la figura 3. Los cambios a lo largo de la serie de datos del total de la red carretera y de las unidades vehiculares de carga provocan un comportamiento similar en las importaciones mexicanas provenientes de Canadá, incluso presentan una tendencia muy parecida en los dos tipos de elasticidades referentes a infraestructura. Se obtuvieron elasticidades positivas en 1996 y 2004, de la misma manera que en la gráfica anterior, aunque con una tendencia mucho más marcada, así como elasticidades negativas para 2009. Dicho comportamiento acentúa la influencia de las crisis económicas en el comportamiento del comercio exterior mexicano.

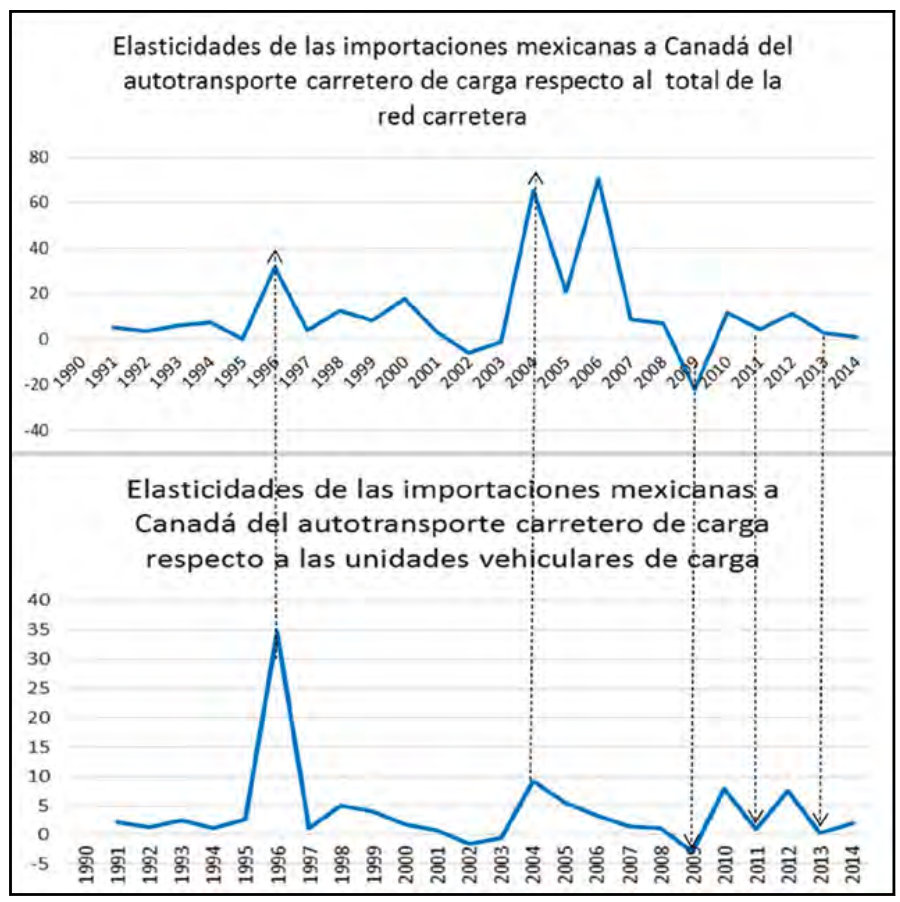

Figura 3. Elasticidades de las importaciones mexicanas de Canadá mediante el transporte carretero con respecto a la infraestructura.

Elaboración propia con base en datos del INEGI (2016) y Estadísticas de Transporte de América del Norte (2013).

En relación con el comportamiento de elasticidades de las exportaciones mexicanas hacia Canadá mediante el transporte carretero con respecto al índice de precios al productor se obtuvo un comportamiento positivo y verdaderamente alto en 2007 y un repunte en 2009 (figura 4). Después las elasticidades comenzaron a disminuir hasta tocar puntos negativos, donde se aprecia una difícil recuperación de las exportaciones, sobre todo si se toma en cuenta que debido al aumento en el diésel 


\section{IIVESTIGAGIÓn Y CUERCIA DE LA UחIVERSIDAD AUTÓNOMA

el precio del transporte de carga mexicano no bajará a corto plazo.

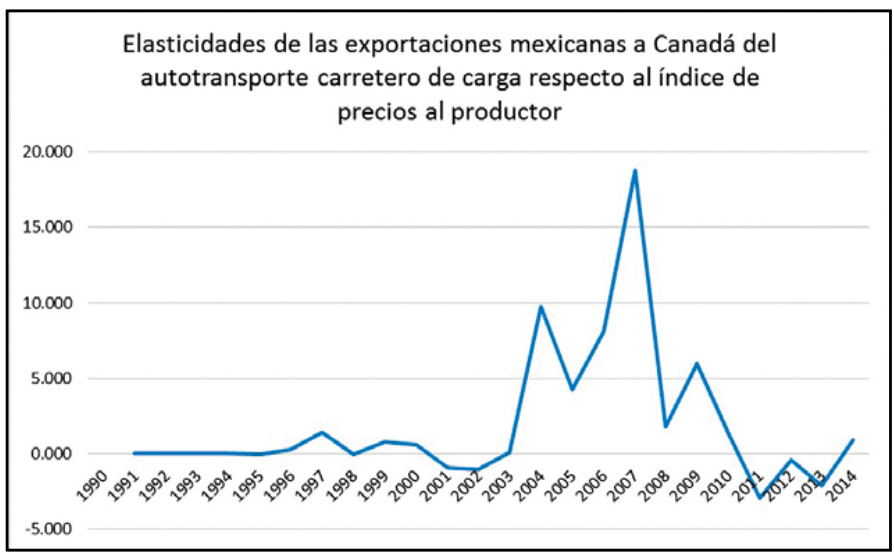

Figura 4. Elasticidades de las exportaciones mexicanas hacia Canadá mediante el transporte carretero con respecto al índice de precios al productor.

Elaboración propia con base en datos del INEGI (2016) y Estadísticas de Transporte de América del Norte (2013).

Por parte de las exportaciones mexicanas hacia Canadá, la infraestructura representada por el total de la red carretera y las unidades vehiculares muestran un comportamiento positivo en las elasticidades calculadas, mismo que puede observarse en la figura 5. Se pueden distinguir elasticidades con valores positivos muy altos en ambos, por señalar algunos se pueden observar los años 2004 y 2009, así como el periodo comprendido desde 1995 hasta 1998, en los cuales aunque se obtuvieron elasticidades positivas para los dos casos, se infiere que las exportaciones mexicanas hacia Canadá son mucho más sensibles a la variación en el número de unidades vehiculares de carga, lo que provoca un cambio mayor. Es importante señalar que en 2004 se inició el programa de chatarrización para las unidades de carga en México y vigente en la actualidad. La mayor parte de las elasticidades obtenidas fueron positivas, aunque es importante destacar el comportamiento drástico de las negativas; estas son observables en 2001 y 2009, años en los que se presentaron dificultades económicas importantes, tanto en México como en otras economías.

Por otra parte, en el año 2000 hay un aumento en el comercio exterior mexicano debido al rápido aumento en las exportaciones a nivel mundial de productos agropecuarios, en la industria extractiva, así como en la producción manufacturera, lo anterior se vio reflejado mayormente en las economías de América del Norte y Asia (Organización Mundial del Comercio, 2002).

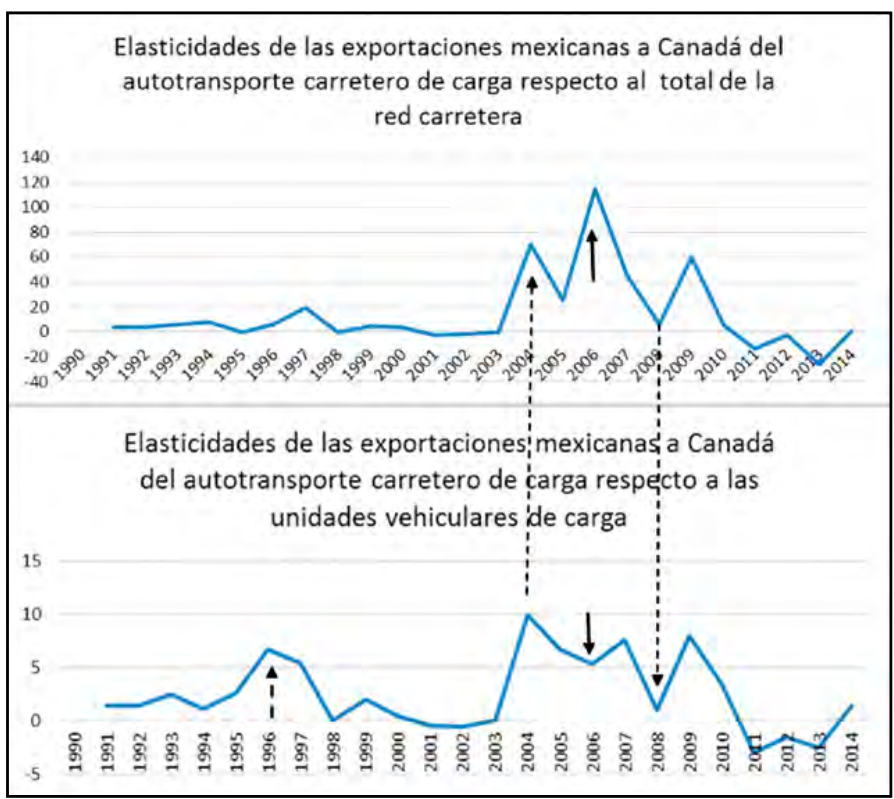

Figura 5. Elasticidades de las exportaciones mexicanas hacia Canadá mediante el transporte carretero con respecto a la infraestructura.

Elaboración propia con base en datos del INEGI (2016) y Estadísticas de Transporte de América del Norte (2013).

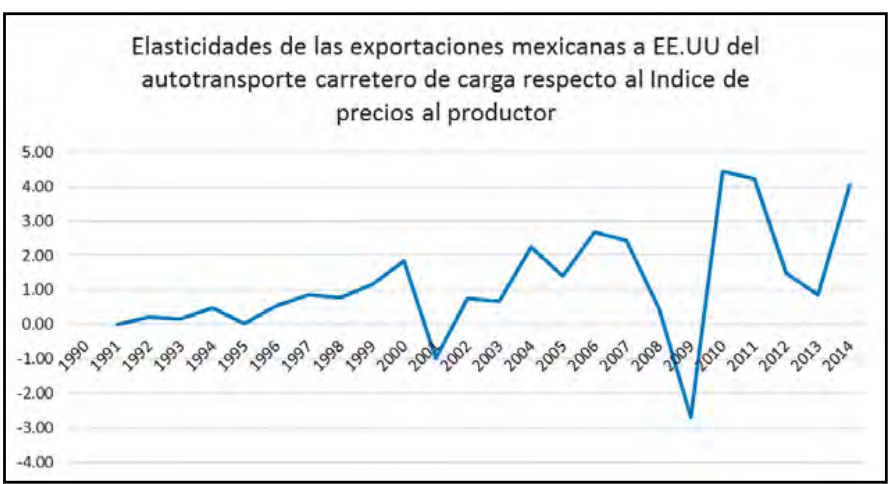

Figura 6. Elasticidades de las exportaciones mexicanas hacia EE. UU. mediante el transporte carretero con respecto al índice de precios al productor.

Elaboración propia con base en datos del INEGI (2016) y Estadísticas de Transporte de América del Norte (2013).

Las elasticidades que se obtuvieron de las exportaciones mexicanas a EE. UU. ante cambios en la red carretera y las unidades vehiculares se pueden observar en la figura 7, presentándose en su mayoría positivas con algunas conductas muy marcadas, sobre todo en 1994, esto no puede explicarse mediante el TLCAN debido a que es el año de su entrada en vigor; aunque la disminución en la elasticidad obtenida de 1995 es claramente un reflejo del efecto tequila generado a finales de 1994. 


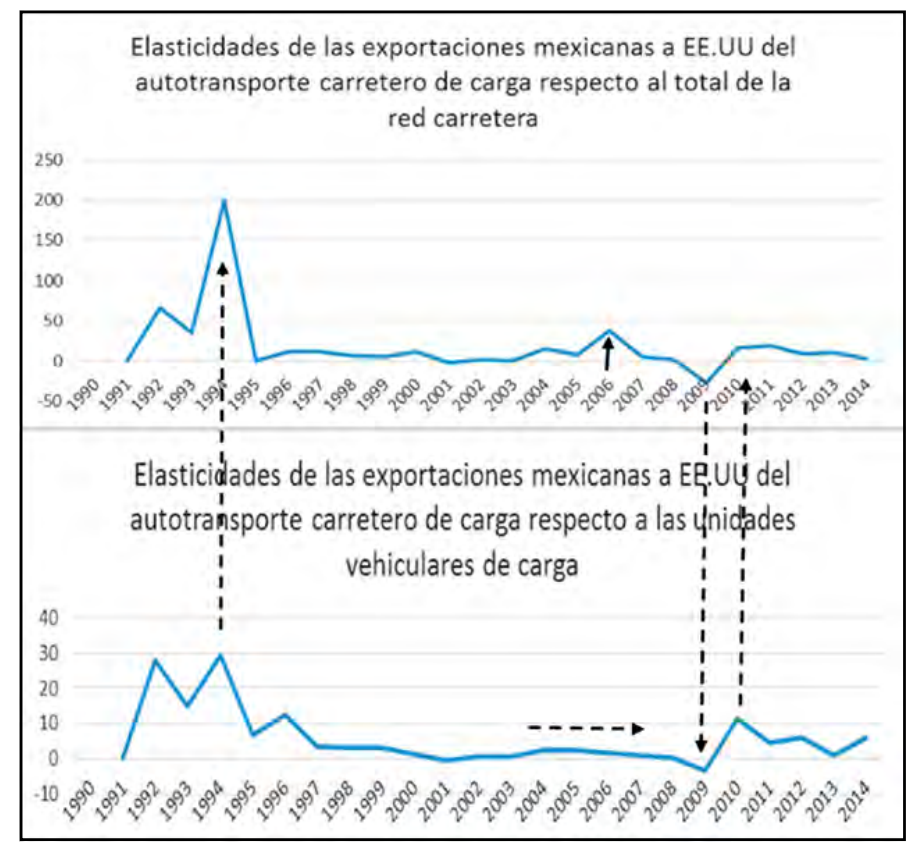

Figura 7. Elasticidades de las exportaciones mexicanas hacia EE. UU. mediante el transporte carretero con respecto a la infraestructura.

Elaboración propia con base en datos del INEGI (2016) y Estadísticas de Transporte de América del Norte (2013).

Por otra parte, en 2006 el aumento de las exportaciones mexicanas hacia EE. UU. fue mayor que en años anteriores, aspecto reflejado en el aumento de la elasticidad obtenida para ese año, sobre todo en los cálculos que incluyen al total de la red carretera, ya que la inversión para esta disminuyó precisamente en 2006, debido al final del sexenio presidencial. En 2009 se obtuvo elasticidad negativa debido a una bastante significativa disminución en las exportaciones.

En relación con las elasticidades obtenidas por parte de las importaciones mexicanas provenientes de EE. UU. con respecto al índice de precios, en 2001 comenzaron a disminuir las importaciones; la caída en ese año fue de $13 \%$, por lo que se obtuvo una elasticidad negativa también debido a que el aumento en el índice de precios no fue suficiente para equilibrar el efecto negativo de las importaciones. Lo anterior se puede observar en la figura 8. La recesión de 2009 también influyó en la disminución de las importaciones para este año, ya que se obtuvo una elasticidad negativa como resultado de la disminución de las importaciones en $23 \%$.

En la figura 9 se puede observar la infraestructura del transporte de carga en México, para las

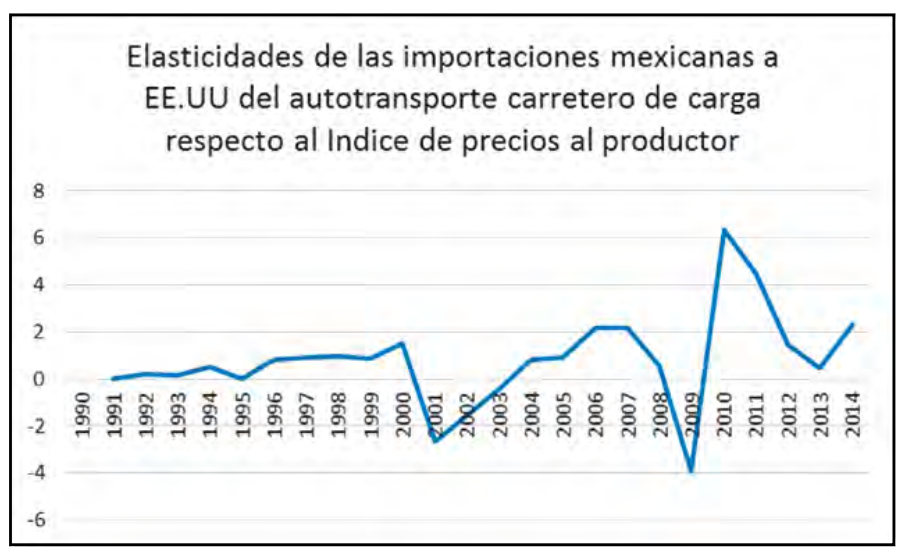

Figura 8. Elasticidades de las importaciones mexicanas de EE. UU. mediante el transporte carretero con respecto al índice de precios al productor.

Elaboración propia con base en datos del INEGI (2016) y Estadísticas de Transporte de América del Norte (2013).

dos series de datos elegidas presenta una influencia muy parecida en las importaciones mexicanas provenientes de EE. UU., ya que las elasticidades positivas y con mayor valor se presentan en los mismos

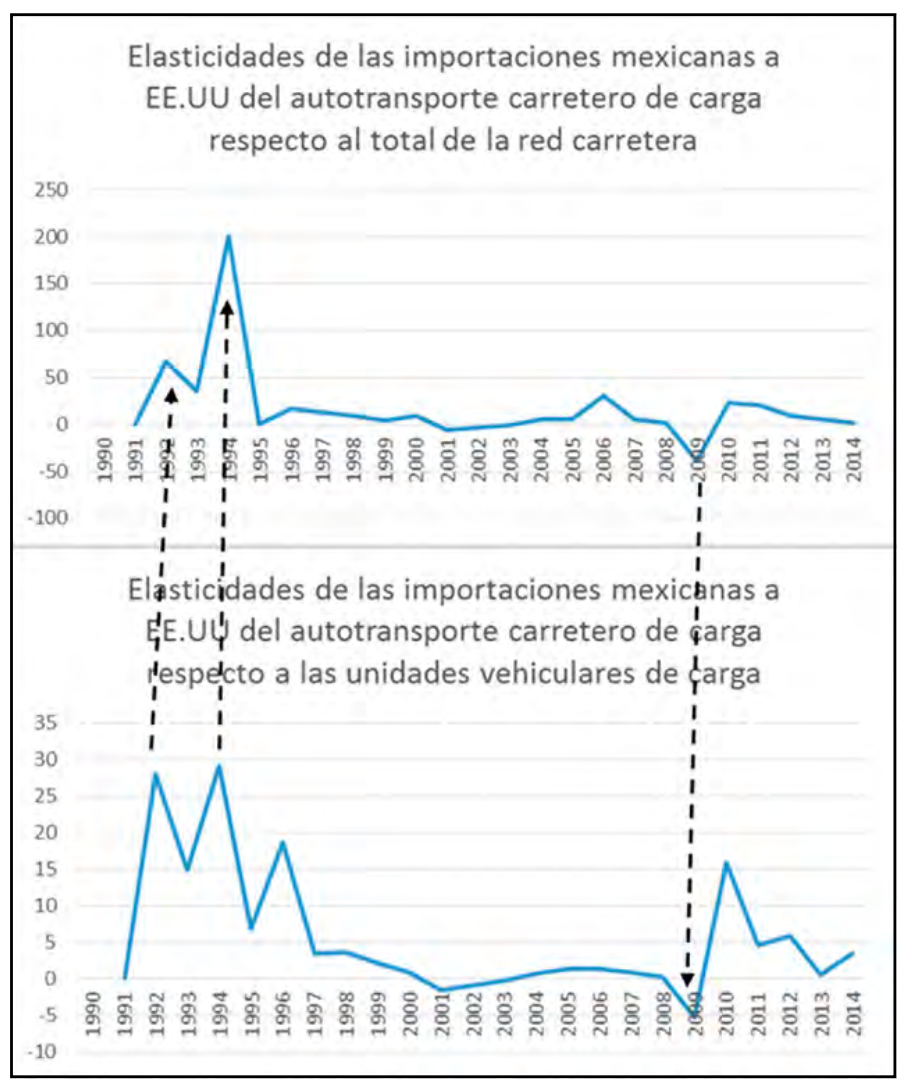

Figura 9. Elasticidades de las importaciones mexicanas de EE. UU. mediante el transporte carretero con respecto a la infraestructura.

Elaboración propia con base en datos del INEGI (2016) y Estadísticas de Transporte de América del Norte (2013). 


\section{InVESTIGACIÓn Y CIERCIR DE LA UNIVERSIDAD AUTÓnOMF DE RGUASCALIERTES}

años; es decir, 1992, 1994 y 1996, aunque resalta la elasticidad negativa obtenida en 2009, que como ya se mencionó es resultado de la disminución de las importaciones en $23 \%$, como consecuencia de la recesión económica.

El cálculo de las elasticidades del volumen de carga mexicano para el mismo periodo, comprendido desde 1990 hasta 2014, se puede observar en la figura 10, en donde resalta el comportamiento positivo de 2007, que en este caso se explica por el aumento en volumen de carga para este año respecto al anterior, de $6.40 \%$.

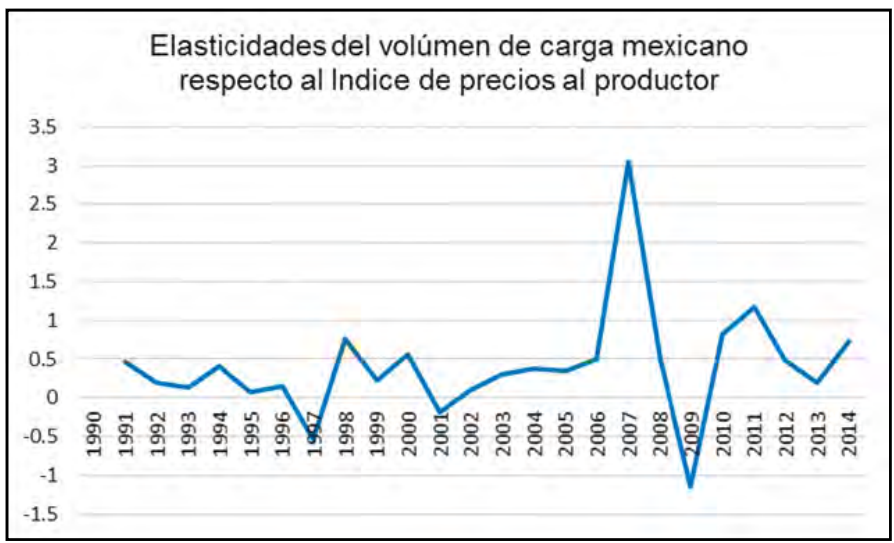

Figura 10. Elasticidades del volumen de carga mexicano con respecto al índice de precios al productor.

Elaboración propia con base en datos del INEGI (2016) y Estadísticas de Transporte de América del Norte (2013).

Pudo haberse tratado de un impulso económico al sector de transporte de carga que no se vio capitalizado como consecuencia de la crisis financiera internacional presentada en 2007, misma que generó que la demanda del mercado estadounidense se paralizara, lo que afectó de manera directa a la economía mexicana. Finalmente, se presentan en la figura 11 las elasticidades calculadas del volumen de carga ante las variaciones de infraestructura.

Como se puede apreciar el comportamiento es en extremo similar, únicamente por la presencia de una elasticidad mucho más marcada en 1994 para el total de la red carretera, explicada por un aumento en el volumen de carga de $2.47 \%$ con respecto al año anterior que, aun cuando no se trata de un incremento significativo, la elasticidad tan alta para este año es consecuencia de la red carretera, que justo para 1994 no creció más que 5 km.

El resto de los resultados presentan elasticidades entre 0 y 1 , lo que podría traducirse en un com-

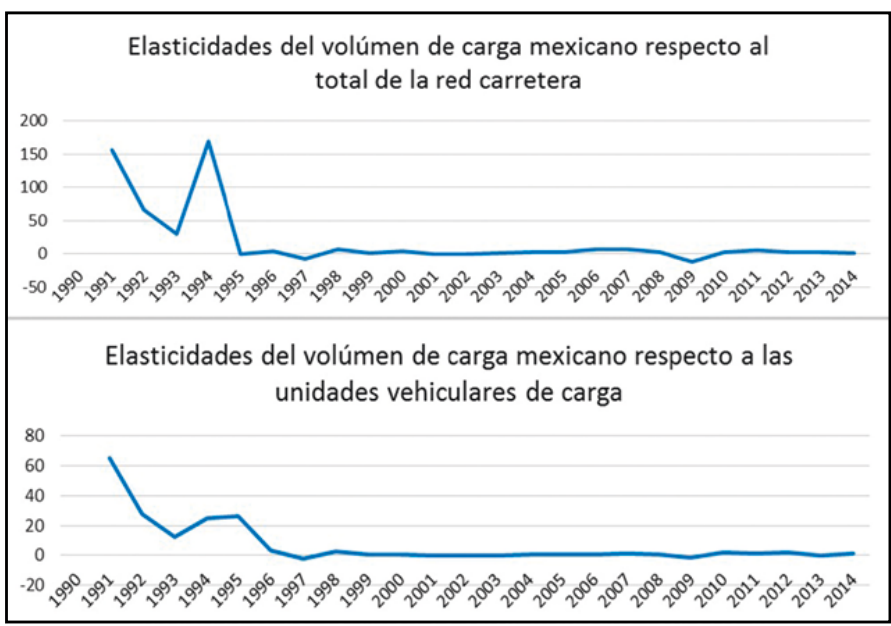

Figura 11 . Elasticidades del volumen de carga mexicano con respecto a la infraestructura.

Elaboración propia con base en datos del INEGI (2016) y Estadísticas de Transporte de América del Norte (2013).

portamiento inelástico; es decir, que el volumen de carga presenta muy poca sensibilidad ante cambios en la infraestructura.

En relación con el resultado del modelo de regresión, las relaciones entre variables son positivas con 99\% de confianza; siendo el índice de precios al productor la variable más influyente en el comercio con Canadá y con EE. UU., aunque también la red carretera provoca una sensibilidad importante en el comercio mexicano con EE. UU.

Tabla 3

Resultados del Modelo de regresión 1990-2014

\begin{tabular}{ccc}
\hline \multicolumn{4}{c}{ Exportaciones a EE. UU.-Transporte carretero de carga } \\
\hline \multicolumn{4}{c}{ RC-Red carretera } & 0.991544 & ${ }^{* *}$ \\
UVC-Unidades vehiculares de carga & 0.02272 & ${ }^{* *}$ \\
IPP-Índice de precios al productor & 0.732356 & ${ }^{* *}$ \\
\hline \multicolumn{4}{c}{ Importaciones a EE. UU.-Transporte carretero de carga } \\
\hline RC-Red carretera & 0.48947 & ${ }^{* *}$ \\
UVC-Unidades vehiculares de carga & 0.010247 & $* *$ \\
IPP-Índice de precios al productor & 0.540834 & $* *$ \\
\hline Exportaciones a Canadá-Transporte carretero de carga \\
\hline RC-Red carretera & 0.027874 & $* *$ \\
UVC-Unidades vehiculares de carga & 0.000728 & $* *$ \\
IPP-Índice de precios al productor & 1.030218 & $* *$ \\
\hline Importaciones a Canadá-Transporte carretero de carga \\
\hline \multicolumn{4}{c}{ RC-Red carretera } & 0.031914 & $* *$ \\
UVC-Unidades vehiculares de carga & 0.000768 & $* *$ \\
IPP-Índice de precios al productor & 1.09871 & $* *$ \\
\hline Nota: Elaboración propia.
\end{tabular}


IIVESTIGACIÓn Y CIERCIA DE LA UחIVERSIDAD AUTÓnOMH DE RGUASCALIERTES
Es importante reflexionar acerca de la verdadera aportación del TLCAN en el contexto del transporte de carga, ya que fue justo después de más de dos décadas de la firma del mismo que las unidades mexicanas tuvieron acceso a territorio estadounidense, por lo que es cuestionable el beneficio de dicho tratado a la fecha en el subsector transporte carretero de carga, ya que las unidades mexicanas no han podido capitalizar la oportunidad planteada a partir de la firma del tratado. Asimismo, se encuentra la particular formación de la estructura empresarial del sector carretero mexicano, mismo que se encuentra conformado por más de 147,000 unidades económicas, de las cuales 128,000 se encuentran en la modalidad hombre-camión y 19,000 como empresas (Ramírez, 2015). Dicha modalidad describe a las microempresas que poseen menos de cinco vehículos, que laboran en la informalidad y representan $81.7 \%$ del total de la estructura empresarial del autotransporte de carga mexicano (SCT, 2013).

En Canadá y EE. UU. la estructura empresarial está conformada en su mayoría por empresas legalmente establecidas, aunque en Canadá existe una figura parecida denominada owners-operators que abarca $25 \%$ de la operación total (Government of Canada, 2016). En los tres países el tamaño de la flota es muy distinta. En 2011 , de acuerdo con las Estadísticas de Transporte de América del Norte, EE. UU. tenía registradas 10.9 millones de unidades, mientras México contaba con 689,000 unidades registradas entre empresas y hombres-camión. Ese mismo año Canadá tenía una flota total registrada de 650,000 unidades; es decir, $5.6 \%$ menos en relación con la registrada en México (ETAN, 2013).

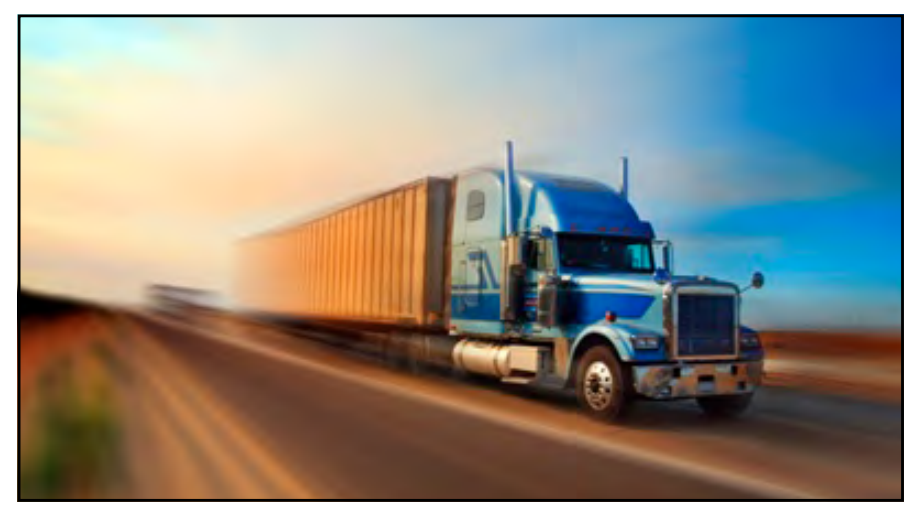

Figura 12. La antigüedad de las unidades afecta la eficiencia operacional y ambiental.

Imagen tomada del portal electrónico AltoNivel.com.mx (2016).
La edad promedio de la flota vehicular también es distinta para los tres países; en México es de 16.5 años, mientras para EE. UU. es de cinco años, muy parecida a la canadiense, con 5.1 años promedio (SCT, 2013). Lo anterior es trascendental debido a que la antigüedad de las unidades afecta la eficiencia operacional y ambiental (figura 12), aumenta el costo del autotransporte por sí mismo debido a los gastos de mantenimiento y genera un aumento en el gasto en combustible, lo que se refleja en el precio final.

En términos de costos y regulación otro factor a considerar es la diferencia en el precio de los combustibles. En 2015 el costo del diésel mexicano era $20 \%$ inferior al estadounidense, lo cual representaba una ventaja en el cálculo del costo del transportista mexicano. Dicha ventaja ha desaparecido debido a los aumentos graduales en el precio de las gasolinas y el diésel como consecuencia de la liberalización de los precios por parte del gobierno mexicano, lo que provoca que actualmente el costo del diésel en México sea mayor al estadounidense en $28 \%$, ello representa una desventaja competitiva importante para el transportista mexicano, ya que al parecer la única ventaja en costos que tienen estos transportistas en la actualidad es que el salario de sus operarios es cinco veces menor que el de un operario estadounidense (Canadian truckers, 2015).

La diferencia de operación de las unidades mexicanas y canadienses con respecto a las estadounidenses se refiere al tonelaje, ya que mientras en Canadá y México se permite que las unidades carguen hasta $25 \mathrm{Tm}$, EE. UU. tiene un límite de carga de $20 \mathrm{Tm}$ aproximadamente (Harrington, 2013). Las unidades mexicanas cargan más debido al doble remolque. Existen varias asociaciones en Méxi$\mathrm{co}$, entre ellas la Alianza Mexicana de Organizaciones de Transportistas, que han estado solicitando la prohibición de este tipo de unidades, debido a la inseguridad que representan.

Por otra parte, en EE. UU. el principal medio de transporte utilizado en el comercio exterior es el marítimo con 47\% según información de 2013, mientras el carretero se emplea en $17 \%$, de acuerdo con datos de North American Transportation Statistics (ETAN, 2013). Debido a la cercanía y a que la mayor parte de las exportaciones mexicanas son dirigidas a EE. UU., el transporte terrestre vía carretera ha mostrado un aumento sostenido en los últimos años, a excepción del año 2009, donde el comercio exterior 
se vio disminuido debido a la recesión provocada por la crisis estadounidense.

La importancia de la variable infraestructura radica en que el incremento en este rubro de cualquier economía, desarrollada o no, permite la ampliación de la operativa entre los mercados y la disminución de los costos de producción. En bastantes estudios generados desde la década de los ochenta se analiza la relación entre inversión en infraestructura y crecimiento económico, tema importante de discusión entre economistas y políticos desde entonces. Esta relación no es nueva, existen autores que han abordado esta relación y han obtenido un comportamiento positivo entre estas dos variables, así como la evidencia de que las economías cuyo gasto público en infraestructura es bajo obtienen un crecimiento económico también bajo. En países como Chile o Perú la inversión en infraestructura carretera se ha llevado a cabo de una manera constante, transparente y sobre todo cumpliendo con los tiempos pactados en el contrato; situación que no se ha impulsado en México en ninguno de los tres aspectos.

La otra variable fue el índice de precios al productor, que en el periodo analizado ha mostrado un incremento constante y lógico de acuerdo con la tendencia inflacionaria anual. Las empresas mexicanas cuyo objetivo es competir con empresas estadounidenses o canadienses hacen énfasis en la diferencia en costos y precios. En relación con la renegociación actual del TLCAN y de la predilección del mandatario actual estadounidense por los acuerdos bilaterales es importante no dejar de lado que dichas acciones han acercado comercialmente a México y China; lo anterior provocado entre otros factores por la salida de EE. UU. del Tratado de Asociación Transpacífico.

Cabe hacer referencia a que gran parte del proceso de desintegración que actualmente se vive con la renegociación del TLCAN se debe en gran medida a la cada vez más predominante presencia de China en el mercado estadounidense, no solamente por aumento de las importaciones y exportaciones entre EE. UU. y China, sino también por una fuerte presencia en el proceso manufacturero y de inversión. Lo anterior pone de manifiesto la importancia de un proceso de diversificación de mercados, además de la renegociación del TLCAN a favor de México. Es indispensable el compromiso del gobierno mexicano para apoyar a las empresas y a los hombres-camión en la capacitación de los requeri- mientos estadounidenses, ya que después de todo, dichos requerimientos no son imposibles de cumplir, sobre todo cuando de versatilidad empresarial se trata.

\section{CONCLUSIONES}

Después de analizar los aspectos más importantes en la operación y regulación de los tres países miembros del TLCAN, las conclusiones del presente son las siguientes:

- Las importaciones mexicanas provenientes de Canadá por medio del transporte carretero son elásticas con respecto al índice de precios al producto; es decir, dichas importaciones sí son sensibles ante las variaciones del índice de precios.

- El comportamiento de dichas importaciones se ha visto afectado de manera directa por crisis internacionales y nacionales como las de 2002 y 2009. En esta relación de variables también se observa un aumento en el consumo de las importaciones canadienses en México, situación que se observa de manera posterior a la firma del TLCAN.

- Las importaciones mexicanas de Canadá mediante el transporte carretero con respecto a la infraestructura presentan un comportamiento elástico y bastante similar entre el total de la red carretera y el número de unidades vehiculares. Es importante mencionar que dichas importaciones presentan un comportamiento positivo que se observa claramente después de la firma del TLCAN.

- Las exportaciones mexicanas a Canadá por medio del transporte carretero en relación con el índice de precios al productor de transporte de carga presenta un comportamiento elástico positivo en mayor magnitud para los años 1997, 2004, 2007 y 2009.

- Se presenta el mismo comportamiento, aunque en diferente medida, para las exportaciones de México hacia Canadá en relación con el total de la red carretera; en esta relación se obtuvo una elasticidad elástica, enfatizando justo en los mismos años que el calculado para el índice de precios.

- En relación con las exportaciones mexicanas hacia Canadá, se puede afirmar que son mucho más sensibles a la variación en el número de unidades vehiculares de carga, provocando un cambio mayor pero siguiendo la misma tendencia que la red carreta o el índice de precios.

- Por otra parte, se calcularon las elasticidades de las exportaciones mexicanas hacia EE. UU. con 
respecto al índice de precios al productor, donde se obtuvo una relación elástica en su mayoría, aunque en 2001 y 2009 la relación fue negativa. De modo posterior a la firma del TLCAN sí se observa una mayor operación comercial en relación con las exportaciones mexicanas hacia EE. UU.

- Con respecto al cálculo realizado para las exportaciones mexicanas hacia EE. UU. en relación con la infraestructura, en su mayoría resultaron elasticidades positivas y con un comportamiento muy similar entre las series de datos utilizadas para representar a la infraestructura; aunque se afirma que los cambios en la red carretera provocan una mayor sensibilidad en el comportamiento de las exportaciones.

- Las elasticidades obtenidas para las importaciones mexicanas provenientes de EE. UU. con respecto al índice de precios presentan resultados sumamente variables, aunque no como consecuencia de los cambios que ha tenido el índice de precios, sino de los cambios que han surgido a través del tiempo en relación con las importaciones mexicanas originarias de EE. UU. Las importaciones mexicanas de origen estadounidense en cuanto a infraestructura son elásticas positivas con excepción de los años 2001 y 2009.

- El volumen de carga mexicano reflejó un comportamiento elástico con respecto a los cambios del índice de precios, con un comportamiento bastante activo y variante; mientras que la misma serie de datos correspondiente al volumen de carga mexicano resultó inelástico respecto a la infraestructura; es decir, presenta muy poca o nula sensibilidad ante cambios en la red carretera y al parque vehicular.

- A grandes rasgos se puede observar que el comercio mexicano con EE. UU. y Canadá efectivamente se volvió más dinámico, lo cual puede ser visto desde un enfoque positivo o negativo, ya que como resultado la economía mexicana también se tornó dependiente de economías internacionales; la prueba se observa en la obtención de grandes variaciones positivas y negativas del comercio exterior mexicano en los años de crisis, tanto internas como externas, que van desde crisis estadounidenses hasta asiáticas. Por ello, la dependencia económica de México no radica únicamente en que la mayoría del comercio exterior sea con EE. UU., sino también a la vecindad que tiene con este país. No obstante, dados los cambios que se aproximan con la renegociación del TLCAN y el constante incremento del comercio de EE. UU. con China, cabe esperar una disminución del flujo comercial con México, el cual no necesariamente percibirá un efecto negativo si comienza a diversificar su comercio exterior. 
REFERENCIAS

- Alto Nivel (2016). [Fotografía]. Recuperada de https://www. altonivel.com.mx/como-los-seguros-pueden-bajar-el-robo-altransporte-de-carga-56829/

- Arvis, J. F., Alina Mustra, M., Panzer, J., Ojala, L., \& Naula, T. (2007). Connecting to compete 2007-Trade Logistics in the Global Economy-The Logistics Performance Index and its indicators. Washington, DC: World Bank.

- Aschaver, D. (1989). Public invesment and productivity growth in the Group of Seven. Economic Perspectives, 13(5), 17-25.

- Cipoletta Tomassian, C., Pérez Salas, G., \& Sánchez, R. J. (2010). Políticas integradas de infraestructura, transporte y logística: Experiencias internacionales y propuestas iniciales. Santiago de Chile: CEPAL.

- Coase, R. H. (1988). The firm, the market and the law. Chicago: The University of Chicago Press.

- Cullen, D. (14 de diciembre de 2015). Trucking inside Mexico. HDT Trucking info [Nota de periódico electrónico]. Recuperado de www.truckinginfo.com

- Daniels, J. D., Radebaugh, L. H., \& Sullivan, D. P. (2010). Negocios internacionales-Ambientes y operaciones. México: Pearson.

- Dorta González, P. (2013). Transporte y logística internacional. Las Palmas, Gran Canaria: Universidad de Las Palmas de Gran Canaria.

- Dussel Peters, E. (2008). Los costos de transporte en las exportaciones mexicanas (Int working paper 13). En M. Mesquita Moreira, C. Bolpe, \& J. S. Blyde (Autores), Destrabando las arterias: El impacto de los costos de transporte en el comercio de America Latina y el Caribe. Cambridge: Banco Interamericano de Desarrollo-Centro de Estudios Latinoamericanos David Rockefeller, Harvard University.

- Estadísticas de Transporte de América del Norte. (2013). Parque Vehicular [Base de datos]. Recuperado el 13 de noviembre de 2014, de http://nats.sct.gob.mx/ir-a-las-tablas-2/tabla12-parque-vehicular/tabla-12-1-numero-de-vehiculosequipode-transporte/

- Garcia-Milà , T., \& McGuire, T. J. (1992). The contribution of publicly provided inputs to states' economies. Regional Science and Urban Economics, 22, 229-241.

- Gómez Leyja, M. S., \& Hernández Rodríguez, S. C. (1995). Introducción a la economía: Un enfoque aplicado. Edo. de México: McGraw-Hill.

- Government of Canada. (28 de abril de 2016). Fuel efficiency benchmarking in Canada's trucking industry. Recuperado el
31 de diciembre de 2016, de www.nrcan.gc.ca/energy/efficiency/transportation/commercial-vehicles/reports/7607

- Gujarati, D. N., \& Porter, D. C. (2010). Econometría. México: McGraw-Hill.

- Harrington, L. (1 de enero de 2013). U. S. Mexico Trade: Twoway traffic. Inbound Logistics. Recuperado de http://www. inboundlogistics.com/cms/article/us-mexico-trade-two-waytraffic/

- IMT. (12 de febrero de 2015). Cómo calcular tarifas de autotransporte de carga. Transporte. MX. El portal del transporte mexicano [Boletín electrónico]. Recuperado de http://www. transporte.mx/como-calcular-tarifas-de-autotransporte-decarga/

- Instituto Nacional de Estadística y Geografía. (2012). Importaciones y exportaciones según modo de transporte, transporte carretero. Banco de Información Económica [Base de datos]. Recuperado el 1 de abril de 2013, de http://www.inegi.org. $\mathrm{mx} /$ sistemas/bie/ idserPadre $=11000380001000100030$

- Instituto Nacional de Estadística y Geografía. (2013). Importaciones y exportaciones según modo de transporte, transporte carretero. Banco de Información Económica [Base de datos]. Recuperado el 1 de enero de 2014, de http://www.inegi.org. $\mathrm{mx} / \mathrm{sistemas} / \mathrm{bie} /$ ? idserPadre $=11000380$

- Instituto Nacional de Estadística y Geografía. (2015). Balanza comercial de mercancías por países y zonas geográficas. Banco de Información Económica [Base de datos]. Recuperado el 1 de enero de 2016, de http://www.inegi.org.mx/sistemas/bie/?idserPadre $=110003300050037000900100$

- Instituto Nacional de Estadística y Geografía. (2016). Índice Nacional de Precios al Productor. Banco de Información Económica [Base de datos]. Recuperado el 4 de marzo de 2016, de http://www.inegi.org.mx/sistemas/bie/?idserPad re $=111002100010001000100100$

- Macías Macías, A. (2003). Tipo de cambio y paridad del poder de compra en México. Comercio Exterior, 53(9), 820-831.

- Mahmud, S. F., Ullah, A., \& Yucel, E. M. (2004). Testing MarshallLerner condition: A non-parametric approach. Applied Economics Letters, 11 (4), 231-236.

- Medina Ramírez, S. (2011). Apertura fronteriza al transporte de carga mexicano: ¿̇Fin del problema? Comercio Exterior, 62(1), 9-11.

- Mendoza Cota, J. E., \& Díaz, E. (2003). Obstáculos al comercio en el TLCAN: El caso del transporte de carga. Comercio Exterior, 53(12), $1112-1120$. 
- Munell, A. H. (1990). Why has productivity growth declined? Productivity and public investment. New England Economic Review, January/February, 3-22.

- Organización Mundial del Comercio. (2002). Evolución del comercio mundial en 2000 y en el primer semestre de 2001 (pp. 1-20). Ginebra: Autor.

- Prud'homme, R. (2005). Infraestructure and development. En F. Bourguignon, \& B. Pleskovic (Eds.)., Annual World Bank Conference on Development Economics, 2005: Lessons of experience (pp. 153-180). Washington, DC: The World Bank-Oxford University Press.

- Ramírez, D. (29 de enero de 2015). Autotransporte, actividad con el mayor costo operativo de combustible. Directorio de Transporte, Logística y Carga T21. Recuperado de http://t21. com.mx/terrestre/2015/01/29/autotransporte-actividad-mayor-costo-operativo-combustible

- Sáez, S., \& Valdés, J. G. (abril de 1999). Chile y su política comercial "lateral". CEPAL, 67, 81-94.

- Secretaría de Comunicaciones y Transportes. (2013). Programa Sectorial de Comunicaciones y Transportes. En Plan Nacional de Desarrollo 2013-2018. México: Gobierno de la República.

- Secretaría de Comunicaciones y Transportes. (Abril de 2014). Título de Concesión que en Materia Ferroviaria, ha otorgado el Gobierno Federal, por Conducto de la Secretaría de Comunicaciones y Transportes [Cuadro informativo]. Recuperado de http://gaceta.diputados.gob.mx/Gaceta/62/2014/ feb/ConseFerr-20140218.pdf
- Secretaría de Comunicaciones y Transportes. $(11$ de enero de 2015). El Departamento de Transporte de los Estados Unidos anuncia su voluntad de recibir solicitudes de transportistas mexicanos para operaciones de largo recorrido [Comunicado]. Recuperado de http://www.sct.gob.mx/despliega-noticias/article/el-departamento-de-transporte-de-los-estadosunidos-anuncia-su-voluntad-de-recibir-solicitudes-de-tr/

- Secretaría de Economía. (2014). A 10 años de la entrada en vigor del TLCAN (6 pp.) [Documento en pdf]. Recuperado de http://www.economia-snci.gob.mx/sic_php/pages/sala_ prensa/pdfs/1-1-22-040223_TLCANX.pdf

- Silvestre Méndez, J. (1999). Fundamentos de economía. México: McGraw-Hill.

- US Census Bureau. (2015). Trade in Goods with Mexico [Base de datos]. Recuperada de https://www.census.gov/foreigntrade/balance/c2010.html\#1994

- US Department of Labor. (2016). Occupational Employment Statistics [Base de datos]. Recuperado de http://www.bls. gov/oes/current/oes533032.htm

- Wagelndicator Foundation (2016). Misalario.org [Base de datos]. Recuperado de http://www.misalario.org/main/tusalario/comparatusalario

- Wilmsmeier, G., \& Sánchez, R. J. (2009). Los desafíos del sistema de transporte en los países sin litoral de América del Sur. Documento de trabajo 142. Santiago de Chile: CEPAL. 